\title{
Update on Nanotechnology-based Drug Delivery Systems in Cancer Treatment
}

\author{
BENJAMIN N. HO, CLAIRE M. PFEFFER and AMARESHWAR T.K. SINGH \\ Department of Biology, Division of Natural and Social Sciences, Carthage College, Kenosha, WI, U.S.A.
}

\begin{abstract}
The emerging field of nanotechnology meets the demands for innovative approaches in the diagnosis and treatment of cancer. The nanoparticles are biocompatible and biodegradable and are made of a core, a particle that acts as a carrier, and one or more functional groups on the core which target specific sites. Nanotech in drug delivery includes nanodisks, High Density Lipoprotein nanostructures, liposomes, and gold nanoparticles. The fundamental advantages of nanoparticles are: improved delivery of waterinsoluble drugs, targeted delivery, co-delivery of two or more drugs for combination therapy, and visualization of the drug delivery site by combining imaging system and a therapeutic drug. One of the potential applications of nanotechnology is in the treatment of cancer. Conventional methods for cancer treatments have included chemotherapy, surgery, or radiation. Early recognition and treatment of cancer with these approaches is still challenging. Innovative technologies are needed to overcome multidrug resistance, and increase drug localization and efficacy. Application of nanotechnology to cancer biology has brought in a new hope for developing treatment strategies on cancer. In this study, we present a review on the recent advances in nanotechnology-based approaches in cancer treatment.
\end{abstract}

Cancer is the second leading cause of death, following heart diseases (1), killing approximately eight million people $(600,000$ per year) and affecting nearly fourteen million (2).

This article is freely accessible online.

Correspondence to: Amareshwar T.K. Singh, Department of Biology, Division of Natural and Social Sciences, Carthage College, Kenosha, Wisconsin 53140, U.S.A. Tel: +1 2625516327, e-mail: asingh1@carthage.edu

Key Words: Nanodisks, gold nanoparticles, HDL nanostructures, liposomes, nanodiamonds, cancer, review.
The rate at which cancer is emerging is only increasing as time goes on due to such factors as increased pollution, radiation, lack of exercise and a balanced diet, among other variables such as genetics (3). Anyone of these factors can lead to a mutation in the DNA of our cells like oncogenes and develop into cancer. The immortalization and sustainability of individual cells capable of reproducing at astonishing rates, overtake all the healthy functional cells, and eventually lead to death.

The most common types of treatment against cancer include chemotherapy, surgery, radiation, and a combination of any of these treatments. However, there are challenges associated with the traditional treatments - non-specificity, toxicity, etc. The challenge of current drug therapy is the optimization of the pharmacological action of the drug, and the minimization of its toxic side effect. Local concentration of the drug at the cancer sites needs to be high, while at other tissues low to prevent any negative reactions. Application of nanotechnology in cancer treatment has the potential to solve these limitations. Designing nanoparticles loaded with multifunctional drugs, and functionalizing their surfaces with recognition proteins can target specific cancer cells $(4,5)$. The advantages of such targeting include the drug amount needed to achieve a therapeutic effect may be significantly reduced as well as the drug concentration on the cancer site can be increased without any bad effects on healthy cells (6).

Several nanoparticle based drug delivery systems nanodisks, HDL nanostructures, gold nanoparticles, and viral nanoparticles - have shown encouraging results in cancer therapy. Progress has been made in studying the biological features of cancer to enhance the use of nanoparticles overcoming biological barriers, and recognizing cancerous tissue vs. healthy tissue. Looking forward, nanodrugs have great potential in cancer therapy due to their unique properties - minimizing toxicity to healthy cells, overcoming multidrug resistance (MDR), and overcoming poor solubility of anti-cancer drugs. 


\section{Nanodisks - Drug Delivery Nanoparticle Vehicles}

Ryan et al. $(7,8)$ synthesized nanodisk (ND) particles comprised of a disk-shaped phospholipid bilayer. The perimeter of the bilayer is stabilized by apoplipoprotein A1 molecules. The apolipoprotein component defines the disk boundary and serves as a "scaffold" that maintains ND particle integrity. The NDs are encapsulated with any hydrophobic drug molecule. NDs are distinguishable from conventional liposomes or vesicles in that they have scaffold proteins constituting an intrinsic structural element of ND; diameters ranging from $8-20 \mathrm{~nm}$ versus $60-250 \mathrm{~nm}$ for liposomes; and most importantly, unlike liposomes, ND are fully soluble in aqueous media. Building on this technology, Ryan et al. (9) created a chimera based on an apolipoprotein-antibody fusion to direct ND particles to a specific cell surface antigen. Certain B-cell lymphomas including Mantle cell lymphoma (MCL), express CD20 as a cell surface protein (10). Studies have shown that the construction of engineered single chain antibody fragments that retain the antigen specificity and binding activity toward CD20 expressing cells displayed by the parent monoclonal antibody $(11,12)$. Since CD20 is a major surface antigen on MCL cells, it provides a potential site for targeted delivery of cytotoxic agents. The recombinant fusion protein comprised of anti CD20 scFv, a spacer sequence and apoAI have dual functions: (a) formation of stable NDs, and (b) possessing CD20 recognition activity.

We (13) pursued a novel approach to deliver an All trans retinoic acid (ATRA) and curcumin (14) payload to MCL cells in culture. This water insoluble bioactive lipid was stably incorporated into nanoscale lipid particles - the NDs $(15,16)$. We hypothesized that, following cellular uptake and/or liberation of ATRA from ND, this retinoid would interact with intracellular binding proteins and, ultimately, nuclear hormone receptors, leading to target gene transactivation, cell growth arrest and/or apoptosis. Indeed, we found that compared to naked ATRA, ATRA-ND elicited enhanced apoptosis and growth arrest in MCL cells, and that this was mediated by RARs and by generation of reactive oxygen species (ROS) (13).

We then tested to see if targeting through scFv-apoA1ATRA-ND (nanodisks scaffolded with a fusion protein apoA1 and a CD20 chain variable fragment, and encapsulated with ATRA) would enhance apoptotis in MCL cells. Compared to medium alone (untreated control), empty NDs had no effect on the number of apoptotic cells while free or naked ATRA showed a slight increase at $20 \mu \mathrm{M}$. At 10 or $20 \mu \mathrm{M}$ doses, scFv-apoA1-ATRA-ND elicited significant apoptosis compared to ATRA-ND or naked ATRA (Figure 1).

Targeting through scFv-apoA1-ATRA-ND, not only casued apoptosis, but enhanced G1 growth arrest in MCL cells as well. scFv-apoA1-ATRA-ND treatment resulted in

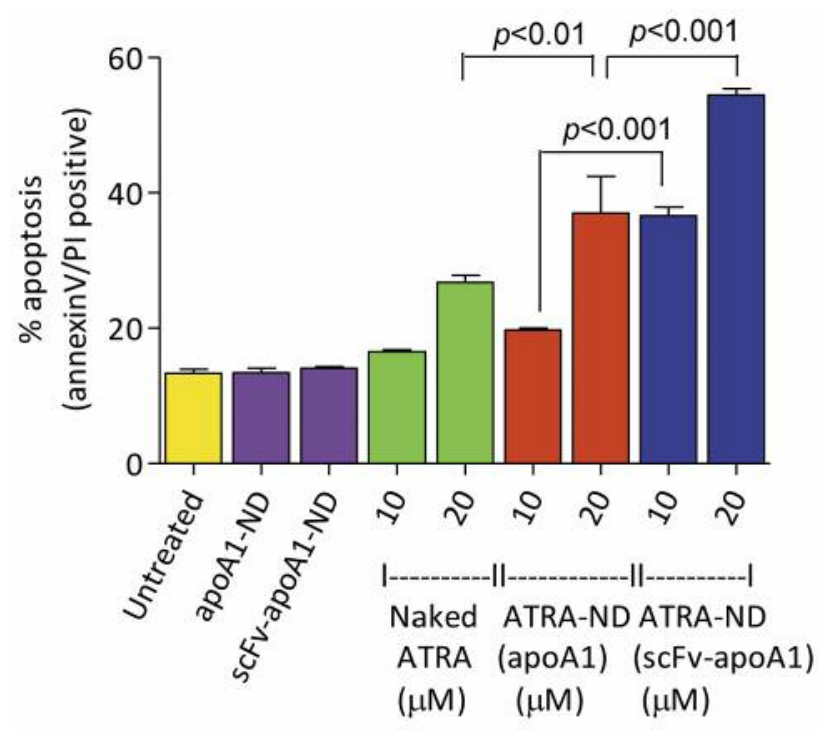

Figure 1. Targeting via scFv-apoA1-ATRA-ND enhanced the apoptotic response in Granta cells. MCL cells (Granta) were treated with medium alone (untreated) empty vehicles (apoAl-ND, scFv-apoAl-ND), naked ATRA, ATRA-ND, and scFv-apoAl-ATRA-ND at $37^{\circ} \mathrm{C}$ in a $5 \% \mathrm{CO}_{2}$ atmosphere. After 48 h, cells were washed in PBS, centrifuged, and resuspended in $100 \mu \mathrm{l}$ binding buffer containing AnnexinV FITC and propidium iodide for $15 \mathrm{~min}$ in the dark. Following $15 \mathrm{~min}$ incubation, $400 \mu \mathrm{L}$ binding buffer was added to each sample prior to flow cytometric analysis. Values shown are the mean $\pm S D(n=3)$. Compared to medium alone (untreated control), empty NDs had no effect on the number of apoptotic cells, while naked ATRA showed a slight increase at $20 \mu \mathrm{M}$. At 10 or $20 \mu \mathrm{M}$ doses, scFv-apoA1-ATRA-ND elicited significant apoptosis compared to ATRA-ND or naked ATRA.

an enhanced G1 arrest compared with non-targeted ATRA$\mathrm{ND}$ and the control groups. There is a progressive decrease in S-phase cells and an increase in G1 growth arrest. More experiments with different doses and time points are being tested to determine statistical correlations within and among different MCL cells (Figure 2).

Later, Crosby et al. (9) demonstrated that the $\alpha$-CD20 scFv-apoA-I-ND bound to CD20-positive non-Hodgkin's lymphoma (NHL) cells (Ramos and Granta) but not to CD20negative T lymphocytes (i.e., Jurkat). Binding to NHL cells was partially inhibited by pre-incubation with rituximab, a monoclonal antibody directed against CD20. Confocal fluorescence microscopy analysis of Granta cells following incubation with $\alpha$-CD20 scFv•apoA-ND formulated with the intrinsically fluorescent hydrophobic polyphenol, curcumin, revealed $\alpha$-CD20 scFv•apoA-I localizes to the cell surface, while curcumin off-loads and gains entry to the cell. Compared to control incubations, viability of cultured NHL cells was decreased upon incubation with $\alpha$-CD20 scFv•apoA-I ND harboring curcumin. 


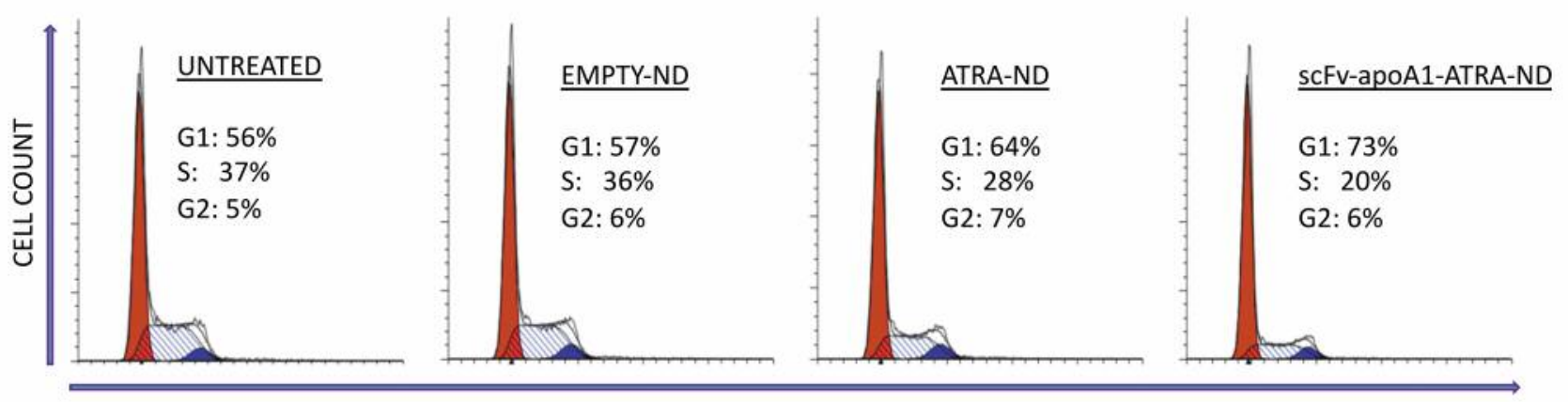

DNA CONTENT

Figure 2. scFv-apoA1-ATRA-ND enhanced $G_{1}$ growth arrest in MCL cells. MCL cells (NCEB) were incubated for 24 hith medium alone (untreated), empty-ND (vehicle control), non-targeted ATRA-ND $(10 \mu M)$ and targeted scFv-apoA1-ATRA-ND (10 $\mu M)$. Following incubation, cells were analyzed for DNA content by Flow Cytometry. The percentage of cells in $G_{1}, S$, and $G_{2}$ were evaluated using ModFit LT for Win32 software (Verity Software House, Topsham, ME). G1 (red), G2 (blue), and $S$ (hatched). scFv-apoA1-ATRA-ND treatment results in enhanced $G_{1}$ arrest compared with nontargeted ATRA-ND and the control groups. There is a progressive decrease in S-phase cells and an increase in $G_{1}$ growth arrest. More experiments with different doses and time points are being tested to determine statistical correlations within and among different MCL cells.

Thus, formulation of curcumin ND with $\alpha-\mathrm{CD} 20 \mathrm{scFv}$ apoA-I as the scaffold component confers cell targeting and enhanced bioactive agent delivery, providing a strategy to minimize toxicity associated with chemotherapeutic agents.

\section{Liposomes}

First discovered by Bangham et al. (17), a liposome is a small biological vesicle composed of amphipathic molecules, like phospholipids, capable of housing any number of materials inside. They vary in size depending on how they are designed but for cancer purposes, range anywhere from 10-200 $\mathrm{nm}$ in diameter (18). These amphipathic molecules form a bilayer in a polar solution so that a vessel is formed. Like a cell, the membranes may contain different types of molecules such as receptors, antibodies, transport proteins, and lipids, among other things (19). They may also be encapsulated with cancer treatment drugs that will be released inside tumor cells and kill them.

The reason nanotechnology such as liposomes can be effective at targeting cancer cells is due to several factors. In a tumor microenvironment, the tumor must initiate angiogenesis to survive. The newly-formed blood vessels are dissimilar to regular arteries and veins in that they are made fairly "unthoroughly". They are more porous because the endothelial cells that compose tumor blood vessels are spaced 600-800 $\mathrm{nm}$ apart making them leaky and less selective to what substances may permeate. This is called the enhanced permeability and retention (EPR) effect (20). By designing liposomes to be less than $600 \mathrm{~nm}$ in diameter, these nanoparticles may be released into tumor sites and not to other parts of the body since normal tissue is bound by tight junctions, preventing the entry of molecules of a certain size. Combined with drugs that induce hypertension (angiotensin-II for example), the effectiveness of nanotechnology can be further improved.

Taking advantage of the EPR effect is not the only way liposomes target cancer cells. The unique quality that these nanostructures have is that, like the plasma membrane, they are able to incorporate nearly anything into their surface. A group of particles that can be referred to as targeting ligands possess the ability to narrow in on chemical markers embedded in cancer cells so they may bind to them. These ligands include aptamers, antibodies, different peptides, and cytokines. Antibodies and aptamers embedded on the outside of liposomes filled with anticancer drugs can actively target cancer cells (18). Doing so allows the drugs to be released inside those cells and only those, leaving all of our healthy cells alone and unaffected. Antibody fragments are even more efficient than whole ones, due to their increased halflives, allowing for more time to seek out their target. Aptamers, on the other hand, are not associated with the immunogenicity of antibodies and prove effective at target specificity of cancer. Being easily eliminated by nucleases after drug delivery they exhibit no adverse toxic effects. While some clinical trials are taking place, there is still research that needs to be done in regards to this method.

With all methods of treatment, there can be side effects. Nanoparticles can be associated with a certain amount of toxicity. This can be due to the relative size, shape, chemical composition, charge, and effects on the immune system. The human body is made up of many different tissues, all of which have pores to allow for the flow of molecules in and out. Vital organs like the spleen will accumulate nanoparticle 
particles larger than $100 \mathrm{~nm}$ while the pores in the liver, being about $100 \mathrm{~nm}$, will aggregate smaller materials (20). Luckily, liposome nanoparticles can be designed to be any size necessary. Those made around $100 \mathrm{~nm}$ wide have been shown to be the optimal size for drug delivery while reducing any toxic effects they may have.

The surrounding environment of the body can also interfere with nanoparticle efficacy. The body's natural fluids, relative $\mathrm{pH}$ and immune system. Polyethylene glycol is a diether, minimally toxic, water soluble chemical that can be used as a protective cover around lipid nanoparticles in order to avoid destruction by phagocytes of the immune system. This coating can prevent the hindrance of the nanoparticles by the human body's natural defense mechanisms and provide a safer route for delivery of treatment (20). It should also be noted that liposomes that are positively charged also exhibit a certain amount of toxicity versus those of negative or neutral quality. By inserting oppositely charged lipids into the nanoparticle, it may be neutralized and eliminate toxicity.

Liposomes are currently in use, delivering chemotherapeutic drugs to tumor microenvironments. The most common of those drugs being doxorubicin and daunorubicin. Researchers have implemented liposomes to tackle some of the more challenging cases in cancer treatment with these two drugs being some of the primary ones used for experimentation. A project conducted by Deng et al. (21) incorporated the use of liposomes to deliver doxorubicin and anti-S100A4 antibodies to breast cancer cells in hope to slow down and halt metastasis, the leading cause of death for those affected. The protein S100A4 is a p53 inhibiting protein that has been seen to prevent p53 from normal tumor suppression. It was found that using modified liposomes as a target specific carrying vessel for doxorubicin and these antibodies, was an efficient route of delivery and that metastasis was successfully inhibited in mice grafts.

\section{High Density Lipoprotein Nanostructures}

Thaxton et al. (22) developed a biomimetic spherical nanoparticle, termed HDL-NP (high density lipoprotein nanoparticle), which has the ability to sequester cholesterol, a feature that is similar to natural HDL. The HDL-NPs were synthesized on a gold nanoparticle (AuNP) on which phospholipids and apolipoprotein A1 were assembled. The AuNP core was enriched with esterified cholesterol which prevented the delivery of cholesterol from HDL-NPs.

B-cell lymphomas constitute a significant segment of Non-Hodgkin lymphomas (23). Lymphoma and other cancers receive cholesterol from HDL for membrane synthesis and proliferation (24). An increased cell proliferation of lymphoma cells was correlated with enhanced esterification of cholesterol, and cell growth was prevented with the inhibition of cholesteryl ester formation (25). Based on this feature of lymphoma cells acquiring HDL cholesterol, we (26) tested targeting of HDL-NPs on lymphoma cells through scavenger receptor type B-1 (SRB1). Our data have shown that HDL-NPs targeted B-cell lymphoma cells through binding to SR-B1 and starved cells from cholesterol (26).

Also, as stated earlier, based on HDL structure, Crosby et al. (9) have built a new technology demonstrating a variety of applications including drug delivery to cancer cells. The nanodisk (ND) technology (27), is a formulation of HDL, and it contains scaffold protein apoA1, as well as a CD20specific single chain variable fragment antibody which binds and specifically delivers hydrophobic anti-cancer drugs to CD20 expressing cells.

\section{Gold Nanoparticles}

Another branch of nanotechnology used in the treatment of cancer are gold nanoparticles (AuNP). Like liposomes, tumor specific antibodies can attach to the surface of the gold particles in order to target cancer cells (18). Taking advantage of the EPR effect after being injected intravenously, the nanoparticles, around $10 \mathrm{~nm}$ wide, congregate in the tumor microenvironment whilst leaving the other tissues alone. The ligands on the gold bind receptors on cancer cells and work their way inside. From there, multiple things can happen. The gold nanoparticles can aggregate together and then a laser of infrared light can be focused on the tumor site. This light energy excites the gold particles, superheating them to the point where gaseous bubbles start to form until the cell can no longer take it and lyse (28). This is quite a physical method of cancer treatment for those tumors that cannot be removed surgically.

Studies being done have shown how AuNP induced hyperthermia in combination with more common therapies can yield positive treatment results (29). This method of tumor reduction has little adverse effects and is minimally toxic with a short half-life so any chemical effects that could occur are rendered inert in mere hours. These researchers do warn, however, that prolonged exposure to the IR laser can potentially start to burn surrounding tissue if not careful though this can be helped by focusing the laser on a small area, thereby minimizing the exposure to other cells. No harmful chemicals are used in AuNPs so long-term damage is not an issue. This form of energy also does not emit any sort of radiation toxic to the human body making it safer and healthier for patients than other sorts of targeted radiation techniques (30).

Gold nanoparticles can also be used as drug delivery systems in a likeness to liposomes. Chemotherapeutic drugs can be conjugated to AuNPs and be used as a means of transport in to cancer cells. In a study done by Tamarkin and Kingston (31), gold nanoparticle (CYT-609) bonded to PEG and TNF was able 
to target tumors ten times more efficiently than just the gold itself and the toxic effects of the compound were lessened. Using a new construct called CYT-21625, which consists of GNP, the paclitaxel substitute TNF and PEG, these scientists tested this nanotech in vivo. Taking advantage of the EPR effect and the tumor targeting capabilities of this compound, CYT21625 was able to release this TNF drug at a steady pace that which had minimal effect on the healthy surrounding tissue. Their findings suggest that a combination of the two treatments would work best in destroying tumors and limit side-effects. The biocompatibility of gold and combined with the protection from the reticuloendothelial system provided by PEGylation of the GNP offers a feasible means of transport for paclitaxel to the tumor microenvironment meaning a high bioavailability.

In addition to gold, the use of other metal nanoparticles is also being studied. These are magnetic nanovectors made-up of cobalt, nickel, or iron that are used in a similar way as gold. Either as a means of drug delivery such as loading vectors with daunorubicin or doxorubicin, or as a way to induce hyperthermia within the tumor (18). To do so requires the focusing of a magnetic field on the tumor site and, just like the GNPs, the magnetic nanoparticles will destroy the cell in which they reside.

\section{Viral Nanodrugs}

The use of viruses as a means of drug delivery has been thrown around in recent years as scientists are quickly realizing how useful viral mechanisms can be. These are naturally-biocompatible nanoparticles that encode their own building instructions using resources from a host cell. Their packaging capabilities are promising when thinking of delivering chemotherapy to tumors. PEGylation can again be used to improve the virus' survivability rate within the immune system and their natural host specificity can be taken advantage of when targeting tumors.

Virus-like particles (VLPs) are constructs that can be designed for just such purposes without worrying about actual viral infection of the entire organism (32). The cowpea mosaic virus has been of interest as of late due to the fact that it has no nucleic acid material inside. It is empty which means that it's use should have no harmful effects as the viral strain does and is therefore safe to be introduced into a living organism. It was found that injecting or inhaling this VLP into a tumor microenvironment could trigger an immune response, releasing large quantities of neutrophils. These recruit cytokines and $\mathrm{T}$ lymphocytes to come in and attack metastatic cancer cells. The results varied from delayed metastasis to complete elimination of the cancer in situ). This method requires no insertion of drug but merely uses our natural defense mechanisms against the cancer. Though no extensive research has been done yet, prospects are high and in development.

\section{Nanodiamonds}

Nanodiamond are microscopic carbonbased agents that have multiple biological applications thanks to the fact that they have very high biocompatibility compared to other nanoparticles of their size. They can be loaded with doxorubicin and carry it to metastatic tumor cells or be used as biomarkers and tracers that label cancer. Chan et al. (33) have used nanodiamonds to not just target cancer cells but once inside, will deliver doxorubicin directly to the mitochondria. This kills the cell be eliminating its power source from the inside and inhibiting growth, reproduction, and regular cell function. By targeting the mitochondria specifically rather than the cell in general, cancerous cells that are typically resistant to doxorubicin, like MCF-7, can still be affected by nanodiamonds.

\section{How Nanotechnological Therapy Compares to that of more Common Treatments?}

The three most common techniques being used today to treat cancer are surgery, radiation, and, of course, chemotherapy. They all involve harsh operations that entail not only killing cancer cells, but regular cells that the body actually needs. Finding alternatives to these methods has been the focus of researches since day one but the problem with most new methods is that they just aren't as effective as what we are already using. How does nanotechnology compare?

From the information that has been gathered, nanoparticle therapies vary in their efficacy and results. There is no consensus because there are so many different kinds of NP treatment techniques. It can be said, however, that most of the research being done has yielded very positive results in the air of killing cancer while leaving healthy tissue alone for the most part. They are associated with far less side effects than that of conventional therapies as the NPs being used are mainly biocompatible, non-toxic, chemically stable materials. Efficient drug delivery means that therapeutic chemicals can be transported safely to target sites without significantly degrading and harming the body. Nanocarriers seem to do just that. Whether or not they are equal to or better than conventional treatments in regards to "treating cancer" is tough to say however further research is needed in order to make this determination. Many researches have actually found that it is beneficial to use a mix of multiple therapies, as is already done in medicine, but incorporating nanocarriers into the mix to offer a wide range of capabilities.

\section{Conclusion and Future Studies}

These forms of nanotechnology and new innovative techniques being used today are some of the most advanced forms of cancer treatment out there. Our research and other studies on 
nanotech-based drug delivery systems used for delivering natural substances such as curcumin, epigallocatechin-3-gallate, and resveratrol on chemotherapeutic approaches against cancer are promising and there is hope that this technology could benefit cancer diagnosis and treatment. Nanocarriers such as liposomes, AuNPs, nanodiamonds, etc. are unique tools to use in order to deliver drugs at a steady controlled pace, without overwhelming the body. In combination with the EPR effect of the tumor microenvironment leaky blood vessels and our ability to specifically target cancer cells with nanoparticles using ligands, antibodies, peptides, and viruses makes this type of therapy a very important topic of interest.

Though the ones listed are only some of the various types of nanotechnologies being studied, further research into how we can use them to our advantage is still moving forward. As it compares to conventional cancer therapies, nanotech seems to be able to stand its ground as some of these new treatments are being used as regular treatments for actual human patients, slowly becoming a norm in oncology. As research continues it is important to take a look at the flaws these nanoparticle treatments may have so they can either be overcome or disregarded to make room for other methods. That being said, prospects for nanotechnology are optimistic at the least and the options for those with cancer who want to get better are ever growing.

There is significant research interest in the drug delivery systems using nanoparticles. Small size, large surface area to mass ratio, and functional structure are the unique features of nanoparticles. These features are explored to various advantages - prolonged circulation time, delivery of drug at the target site, improved bioavailability, and reduced toxicity. Though progress in drug delivery by nanotechnological devices has been dramatic, however, there are some challenges that need to be addressed: (a) deeper understanding of intracellular uptake mechanisms, and the fate of nanodevices in biological tissues, (b) any negative or side-effects of the interactions between nanodevices and biological systems - production of free radicals, damage to the interacting tissue, (c) scaling up of pilot experiments to large scale production, cost of materials needed, quality control for nanodevice materials, and (d) funding support for startup companies to bring the nanodrugs to market. The challenges in targeted drug delivery systems employing nanoparticles can be prevailed over through investigating the restraints on nanoparticle approaches and maximizing the current potential of nanoparticle formulations and devices.

\section{Conflicts of Interest}

The Authors declare no competing financial interests.

\section{Acknowledgements}

The Authors would like to gratefully acknowledge the support from the Department of Biology, Division of Natural and Social Sciences,
Carthage College, Kenosha, WI, USA, for supporting Benjamin N. Ho and Claire M. Pfeffer as Fellows to conduct research under the mentorship of Dr. Amareshwar T.K. Singh.

\section{References}

1 Siegel RL, Miller KD and Jemal A: Cancer statistics CA: A Cancer J Clin 65: 5-29, 2015.

2 Arnold R: Prospective cancer treatment found in the Cowpea Mosaic Virus. Microreviews Cell Mol Biol 1(1), 2016.

3 Anand P, Kunnumakkara AB, Sundaram C, Harikumar KB, Tharakan ST, Lai OS and Aggarwal BB: Cancer is a Preventable Disease that Requires Major Lifestyle Changes. PharmRes 25(9): 2200-2200, 2008.

4 Duncan R: Polymer conjugates as anticancer nanomedicines. Nat Rev Cancer 6: 688-701, 2006.

5 Couvreur $\mathrm{P}$ and Vauthier C: Nanotechnology: Intelligent designto treat complex disease. Pharm Res 23: 1417-1450, 2006.

6 Vasir JK, Reddy MK and Labhasetwar VD: Nanosystems in drug targeting: Opportunities and Challenges. Curr Nanosci 1: 47-64, 2005.

7 Redmond KA, Nguyen TS and Ryan RO: All-trans retinoic acid nanodisks. Int J Pharm 339: 246-250, 2007.

8 Ryan RO: Nanodisks: hydrophobic drug delivery vehicle. Expert Opin Drug Deliv 5: 343-351, 2008.

9 Crosby NM, Ghosh M, Su B, Beckstead JA, Kamei A, Simomsen JB, Luo B, Gordon LI, Forte TM and Ryan RO: Ant-CD20 single chain variable antibody fragment-apoA1 chimera containing nanodisks promote targeted bioactive agent delivery to CD20positive lymphomas. Biochem Cell Biol 93: 343-350, 2015.

10 Witzig TE: Current treatment approaches for mantle-cell lymphoma. J Clin Oncol 23: 6409-6414, 2005.

11 Shan D, Press OW, Tsu TT, Hayden MS and Ledbetter JA: Characterization of $\mathrm{scFv}$-Ig constructs generated from the anti$\mathrm{CD} 20 \mathrm{mAb} 1 \mathrm{~F} 5$ using linker peptides of varying lengths $\mathrm{J}$ Immunol 162: 6589-6595, 1999.

12 Hamdy N, Goustin AS, Desaulniers JP, Li M, Chow CS and AlKatib A: Sheep red blood cells armed with anti-CD20 singlechain variable fragments (scFvs) fused to a glycosylphosphatidylinositol (GPI) anchor: a strategy to target CD20-positive tumor cells. J Immunol Methods 297: 109-124, 2005.

13 Singh ATK, Evens AM, Anderson RJ, Beckstead JA, Sankar N, Sassano A, Bhalla S, Yang S, Platanias LC, Forte TM, Ryan RO and Gordon LI: All trans retinoic acid nanodisks enhance retinoic acid receptor mediated apoptosis and cell cycle arrest in mantle cell lymphoma. Brit J Haematol 150: 158-169, 2010.

14 Stauffer RG, Manar M and Singh ATK: Novel Nanoscale Delivery Particles Encapsulated with Anticancer Drugs, All-trans Retinoic Acid or Curcumin, Enhance Apoptosis in Lymphoma Cells Predominantly Expressing CD20 Antigen. Anticancer Res 35: 6425-6430, 2015.

15 Redmond KA, Nguyen TS, Ryan, RO: All-trans-retinoic acid nanodisks. Int J Pharm 339: 246-250, 2007.

16 Ryan RO: Nanodisks: hydrophobic drug delivery vehicles. Expert Opinion on Drug Delivery 5: 343-351, 2008.

17 Bangham AD: A correlation between surface charge and coagulant action of phospholipids. Nature 192: 1197-1198, 1961.

18 Jabir NR, Tabrez S, Ashraf GM, Shakil S, Damanhouri GA and Kamal MA: Nanotechnology-based approaches in anticancer research. Int J Nanomed 7: 4391-4408, 2012. 
19 Siddiqui IA, Adhami VM, Christopher J and Mukhtar H: Impact of nanotechnology in cancer: emphasis on nanochemoprevention. Int J Nanomed 7: 591-605, 2012.

20 Singh S, Sharma A and Robertson GP: Realizing the Clinical Potential of Cancer Nanotechnology by Minimizing Toxicologic and Targeted Delivery Concerns. Cancer Res 72(22): 5663-5668, 2012.

21 Deng H, Song K, Zhao X, Li Y, Wang F, Zhang J and Qin Z: Tumor Microenvironment Activated Membrane Fusogenic Liposome with Speedy Antibody and Doxorubicin Delivery for Synergistic Treatment of Metastatic Tumors. ACS ApplMater Interfaces 9(11): 9315-9326, 2017.

22 Thaxton CS, Daniel WL, Giljohann DA, Thomas AD and Mirkin CA: Templated specrical high density lipoprotein nanoparticles. J Am Chem Soc 131: 1384-1385, 2009.

23 Hayat MJ, Howlader N, Reichman ME and Edwards BK: Cancer statistics, trends, and multiple primary cancer analyses from the surveillance, epidemiology, and end results (SEER) program. Oncologist 12: 30-37, 2007.

24 Leon CG, Locke JA, Adomat HH, Etinger SL, Twiddy AL, Neumann RD, Nelson CC, Guns ES and Wasan KM: Alterations in cholesterol regulation contribute to the production of intratumoral androgens during progression to castrationresistrant prostate cancer in a mouse xenigraft model. Prostate 70: 390-400, 2010.

25 Mulas MF, Abete C, Pulisci D, Pani A, Massidda B, Dessì S and Mandas A:Cholesterol esters as growth regulators of lymphocytic leukemia cells. Cell Prolif 44: 360-371, 2011.

26 Yang S, Evens AM, Prachand S, Singh AT, Bhalla S, David K and Gordon LI:Mitochondrial-mediated apoptosis in lymphoma cells by the diterpenoid lactone andrographolide, the active component of Andrographis paniculata. Clin Cancer Res 16: 4755-4768, 2010
27 Ryan RO: Nanobiotechnology applications of reconstituted high density lipoprotein. J Nanobiotechnol 8: 28-38, 2010.

$28 \mathrm{Ou}$ Y, Webb JA, Faley S, Shae D, Talbert EM, Lin S and Bardhan, R: Gold Nanoantenna-Mediated Photothermal Drug Delivery from Thermosensitive Liposomes in Breast Cancer. ACS Omega 1(2): 234-243, 2016.

29 Ren Y, Qi H, Chen Q and Ruan L: Thermal dosage investigation for optimal temperature distribution in gold nanoparticle enhanced photothermal therapy. Int J Heat Mass Transfer 106: 212-221, 2017.

30 Ajnai G, Chiu A, Kan T, Cheng C, Tsai T and Chang J: Trends of Gold Nanoparticle-based Drug Delivery System in Cancer Therapy. J Exp Clin Med 6(6): 172-178, 2014.

31 Tamarkin L and Kingston DG: Exposing the tumor microenvironment: how gold nanoparticles enhance and refine drug delivery. Ther Deliv 8(6): 363-366, 2017.

$32 \mathrm{Ma} \mathrm{Y,} \mathrm{Nolte} \mathrm{RJ} \mathrm{and} \mathrm{Cornelissen} \mathrm{JJ:} \mathrm{Virus-based} \mathrm{nanocarriers} \mathrm{for}$ drug delivery. Adv Drug Deliv Rev 64(9): 811-825, 2012.

33 Chan MS, Liu LS, Leung H and Lo PK: Cancer-Cell-Specific Mitochondria-Targeted Drug Delivery by Dual-LigandFunctionalized Nanodiamonds Circumvent Drug Resistance. ACS Appl Mater Interfaces 9(13): 11780-11789, 2017. 